\title{
Effect of Potential Shape on the Density of the Impurity States in Nanotube
}

\author{
Eduardo A. Orozco*, Jesús D. González ${ }^{\dagger}$, and Martha L. Barrera ${ }^{\dagger}$ \\ * Facultad de Ingeniería de Telecomunicaciones, Universidad Santo Tomás, A.A 1076, Bucaramanga, Colombia \\ ${ }^{\dagger}$ Facultad de Ingeniería y ciências Naturales. Universidad Autónoma de Bucaramanga, A.A 1642, Bucaramanga, Colombia
}

Received on 08 December, 2005

\begin{abstract}
We analyze the effect of the potential shape on the ground state energy of the off-axis neutral donor in $G a A s / G a_{1-x} A l_{x} A s$ cylindrical nanotube in the presence of the uniform magnetic field applied along the symmetry axis. To take into account the mixing of the low lying subbands we express the wave function as a product of combination of $1 s$ and $2 p_{x, y}$ wave functions with an unknown envelope function that depends only on electron-ion separation. By using variational principle and the functional derivative procedure we derive a one-dimensional differential equation for the envelope function, which we solve numerically by using of the trigonometric sweep method. Results of calculation of the ground state binding energy dependencies on the distance from the donor position to the axis and on the strength of the external magnetic field for square-well, soft-edge-barrier and parabolic bottom potentials are presented. It is shown that the additional peaks in the curves of the density of impurity states appear due to the presence of the repulsive core is nanotube.
\end{abstract}

Keywords: Subbands mixing; Magnetic field; Density impurity states. Binding energy

\section{INTRODUCTION}

In the last two decades, there has been an increasing interest in the study of the peculiar physical properties of QWWs (Quantum Well Wires). The study of bound impurity states in such Q1D (One dimensional) structures is therefore considered to be a subject of fundamental interest and a significant attention [1]. Extensive theoretical investigation on the behavior of shallow impurities in QWW has been developed using the Bastard type trial function. In nanotubes, which are QWWs with a repulsive core around the wire axis, the confining potential along cross section through the axis of the nanotube is similar to one of a symmetric double quantum well. As it has been demonstrated previously, the inclusion of the subband mixing plays an important role in correctly determining off-center donor binding energies in double quantum well [2]. In our previous work we show that the low lying $1 s$ and $2 p_{x, y}$ subbands of the free electron in nanotube, similarly to the case of a double quantum well, become almost degenerated as the width of the repulsive core grows and the mixing of these subbands in the presence of the off-axis donor no longer should be depreciated [3]. In this work we analyze the ground state energy of the off-axis neutral donor and the density of the impurity states in $\mathrm{GaAs} / \mathrm{Ga}_{1-x} \mathrm{Al}_{x} \mathrm{As}$ cylindrical nanotube with different potential shapes in the presence of the uniform magnetic field applied along the symmetry axis. The trial function of the donor is taken as a product of combination of $1 s$ and $2 p_{x, y}$ subband wave functions with an unknown envelope function that depends only on electron-ion separation. Using the fractal dimension method [4] we find the onedimensional differential equation for the envelope function, which we solve numerically.

\section{THEORY}

Using the effective Bohr radius, $a_{0} *$ and the Rydberg, $R y^{*}$ as a units of length and energy, respectively, and neglecting differences between material parameters in the barrier and in the well, the dielectric constant $\varepsilon$ and the electron effective mass $m *$, the dimensionless Hamiltonian for a neutral donor impurity in a cylindrical nanotube in the presence of an applied uniform magnetic field $\vec{B}=B \hat{z}$ in the effective mass approximation, can be written as:

$$
H=H_{0}-\frac{2}{|\vec{r}-\vec{\xi}|} ; H_{0}=-\nabla^{2}+V(\rho)+\frac{1}{4} \gamma^{2} \rho^{2}-i \gamma \frac{\partial}{\partial \varphi}
$$

where $\vec{r}$ and $\vec{\xi}$ are used to designate the electron and ion positions, respectively. $V(\rho)$ is the confinement potential with axial symmetry and with a repulsive core around the axis of the nanotube and $\gamma=e \hbar B / 2 m * c R y *$ is the magnetic field strength corresponding to the first Landau level expressed in $R y^{*}$.

To calculate the donor ground state energy, we choose a trial function as a product of the linear combination of the waves functions of the electron ground, $s$ and the first excited, $2 p_{x, y}$ states, with an unknown one-dimensional function

$$
\begin{aligned}
& \Phi(|\vec{r}-\vec{\xi}|): \\
& \qquad \psi(\vec{r})=\left[\alpha f_{1 s}+\sqrt{1-\alpha^{2}} f_{2 p}\right] \Phi(|\vec{r}-\vec{\zeta}|),
\end{aligned}
$$

where $\alpha$ is a variational parameter $(-1<\alpha<1)$ which gives the grade of the mixing of the subbands [3]. One can see that in the particular case as $\alpha=1$, there are not subband mixing and the trial function (2) becomes similar to the simple Bastard-type trial function. In general case, the mixing provided a decrease of the parameter $\alpha$. Greater the mixing the smaller is the parameter $\alpha$. In our calculations the parameter $\alpha$ decrease at to 0.8 .

For our model the wave equation for the free electron $H_{o} f(\vec{r})=E_{0} f(\vec{r})$ is separable and the electron wave function can be written as follows:

$$
f(\vec{r})=e^{i k z} e^{i m \varphi} g(\rho)
$$


where $m=0, \pm 1, \pm 2, \ldots$ is the angular momentum in $z$ direction, $k$ is the wave number corresponding to a free motion in $z$-direction $(-\pi<k<\pi)$ and the function $g(\rho)$ is the solution of the one-dimensional boundary value problem:

$$
\begin{aligned}
& g^{\prime \prime}(\rho)+\frac{1}{\rho} g^{\prime}(\rho)+U(\rho) g(\rho)=0 ; g^{\prime}(0)=0 ; g(\infty)=0 \\
& U(\rho)=E_{0}-\gamma m-k^{2}-V(\rho)-\frac{\gamma^{2} \rho^{2}}{4}-\frac{m^{2}}{\rho^{2}}
\end{aligned}
$$

The differential equation (4) is solved numerically using the trigonometric sweep method [5]. In our calculations we use the confinement potential modelling a nanotube given by the following expression:

$$
V(\rho)=V_{i} \theta\left(-\rho,-R_{i}, W\right)+V_{e} \theta\left(\rho, R_{e}, W\right),
$$

where:

$$
\theta\left(z, z_{0}, W\right)=\left\{\begin{array}{cc}
0 & z<z_{0}-W \\
{\left[\left(z-z_{0}\right) / W^{2}-1\right]^{2}} & z-W \leq z<z_{0} \\
1 & z \geq z_{0}
\end{array}\right.
$$

is soft-edge version of the Heaviside function, $R_{i}, V_{i}$ and $R_{e}$, $V_{e}$ represent the radii and heights of the repulsive core and the barrier, respectively; $W$ is a parameter related to the width of the transition region (see Fig. 1). In the particular case, as $R_{i}=0$, the potential (5) describes a cylindrical QWW.

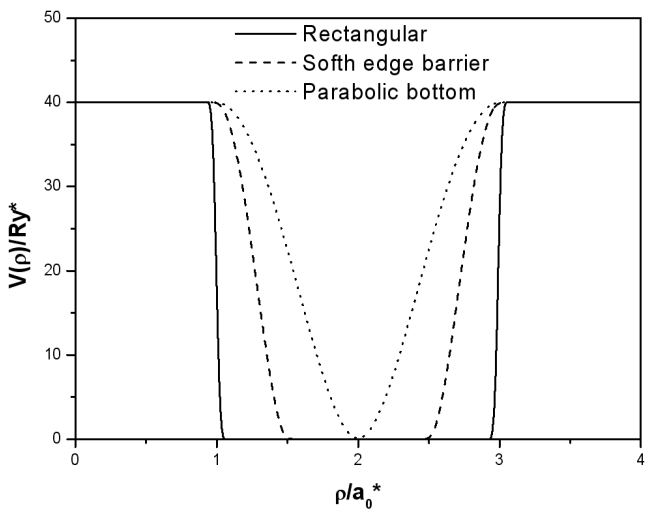

FIG. 1: Confinement Potential in cylindrical Nanotube.

The solutions of the boundary value problem (4) corresponding to the bottom of the subbands $(k=0)$ with radial quantum numbers $n=0,1,2, \ldots$ and the angular momentum $m$. We denote as $g_{n, m}(\rho)$ and therefore in our notations the electron wave function, $f_{n, m}(\vec{r})$ and the energy $E_{0}(n, m)$ depend on two quantum numbers $(n, m)$.

If we assume that the off-center donor is located on the axis $x$, then it modifies the free electron wave functions in such way that it becomes more asymmetric in the $x$ direction. It is reason why in the trial function (2) should be chosen the electron wave functions which gives contribution in the mixing as $f_{1 s}(\vec{r})=g_{0,0}(\rho)$ for the $1 s$ state, and $f_{2 p}(\vec{r})=g_{0,1}(\rho) \cos \varphi$ for $2 p_{x}$ state. Starting from the variational principle and using the method described in the papers [6] one can obtain the following Euler-Lagrange equation for the correlation function:

$$
-\frac{1}{J(r)} \frac{d}{d r}\left[J(r) \frac{d \Phi(r)}{d r}\right]+\left[\tilde{E}(r)-\frac{2}{r}\right] \Phi=E\left(D^{0}\right) \Phi
$$

Where $E\left(D^{0}\right)$ represents the energy of the neutral donor, $J(r)$ is radial part of the Jacobian and $\tilde{E}(r)$ is the averaged free electron local energy, given by the followings expressions:

$$
J(r)=r^{2} \sum_{i, k=0,1} \alpha^{2-i-k}\left(1-\alpha^{2}\right)^{(i+k) / 2} P_{i k}(r)
$$

$$
\tilde{E}(r)=r^{2} \sum_{i, k=0,1} \alpha^{2-i-k}\left(1-\alpha^{2}\right)^{(i+k) / 2} E_{0}(0, i) P_{i k}(r) / J(r)
$$

$$
\begin{aligned}
& P_{i k}(r)=\int_{0}^{2 \pi} \int_{0}^{\pi} g_{0,0}^{2-i-k}(\tilde{\rho}) g_{0,1}^{i+k}(\tilde{\rho}) \lambda^{i+k} \sin \theta d \theta d \varphi ; i, k=0,1 \\
& \lambda=1-r^{2} \sin ^{2} \theta \sin ^{2} \varphi / \tilde{\rho}^{2} \\
& \tilde{\rho}=\left(r^{2} \sin ^{2} \theta+\xi_{\rho}^{2}+2 r \xi_{\rho} \sin \theta \cos \varphi\right)^{1 / 2}
\end{aligned}
$$

In these relations $\xi_{\rho}$ is the distance from the donor position to axis. Once the functions $g_{0,0}(r)$ and $g_{0,1}(r)$ are found, the functions $P_{i, k}(r),(i, k=0,1)$ and $J(r)$ may then be calculated in straightforward way through (7b) and (7d). Finally, to define the donor energy we solve the wave Eq. 6(a) by using the trigonometric sweep method [5]. To calculate the density of the impurity states we assume that the circular cross section of the nanotube is not too small in order that we might treat the impurity positions as a continuous random variable. In this case the density of the impurity states $g(E)$ in a cylindrical nanotube is given by the following relation [4]:

$$
g\left(E_{b}\right)=2 \pi \xi_{\rho}\left(E_{b}\right)\left|d E_{b}\left(\xi_{\rho}\right) / d \xi_{\rho}\right|^{-1}
$$

In our calculations we use the material parameters for $\mathrm{GaAs} / \mathrm{Ga}_{07} \mathrm{Al}_{0.3} \mathrm{As}$ heterostructures given in Refs. [2].

In Fig. 2 we display the results of calculation of the density impurity state in the cylindrical QWW (the repulsive core radius is equal to zero) of the radius $3 a_{0} *$. In the inset we show the dependence of the donor binding energy on the distance from its position to the axis. Different curves in this figure correspond to different magnetic field strength from $\gamma=0$ to $\gamma=3$. In all cases the maximum of the binding energy corresponds to the donor location at the axis of the QWW (see inset). It is seen that the value of the maximum binding energy in the inset rises with increasing of the magnetic field strength. This increase provides a significant displacement of the right-side threshold in the curves of the density impurity states to the region of the larger energies when the magnetic field increases. Also one can observe in the inset the all curves 
intersect at the same point as the distance from the donor location to the axis approach to the value about one Bohr radius and when the separation between the donor and the axis increases further the order of the curves is inverted. It is due to the fact that the electron, under condition of the strong confinement in the radial direction is localized mostly nearly the axis independently of the donor position. Therefore the larger the distance from the donor position to the axis the greater is the separation between the electron and the donor and the smaller is the binding energy. In the presence of the external magnetic field applied along the growth axis the electron becomes more confined and the binding energies of donors located nearly to the axis increase whereas they decrease for peripheral donors. It is the reason why the curves in inset of Fig. 2 intersect and the order of the curves is inverted with the displacement the donor position toward the peripheral region. Also it is seen that for donor positions with the distance from the axis equal one Bohr radius the binding energy doesn't depend on the external magnetic field

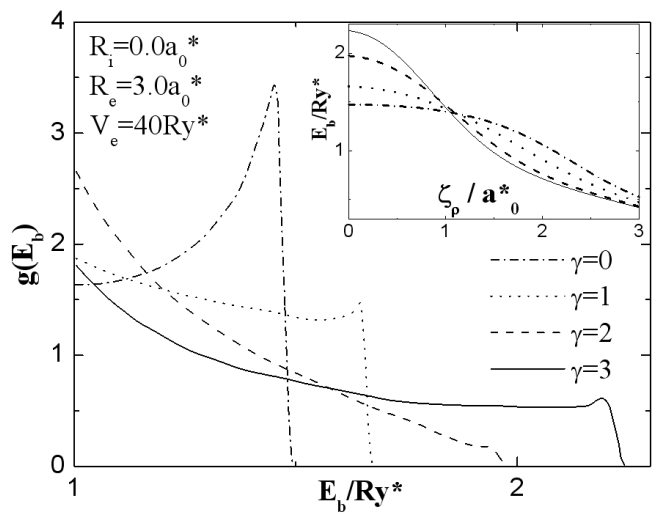

FIG. 2: Density of the $D^{0}$ impurity states as a function of the binding energy in a QWW with rectangular confinement potential for several values of the magnetic field $B$ applied along the symmetry axis. Inset shows the corresponding donor binding energies dependencies on the distance from the axis.

One can see also in Fig. 2 that the density of the impurity states for zero-magnetic-field case has a strongly pronounced peak at the right side threshold point due to the existence of the Van Hove-type singularity at the maximum of the dashdotted curve in the inset. As the strength of the magnetic field increases the singularity in the curves of the binding energy dependence on the donor position disappears, providing the peak dropping and its displacement to the region of the larger energies.

In Fig. 3, We present the $D^{0}$ binding energy dependence on the donor displacement from the center of the nantube for different values of the magnetic field $B$ applied parallel to the axis. One can observe that in absence of the magnetic field the electron cannot penetrate in the central region, it mostly located in the middle between two barriers. If the donor is

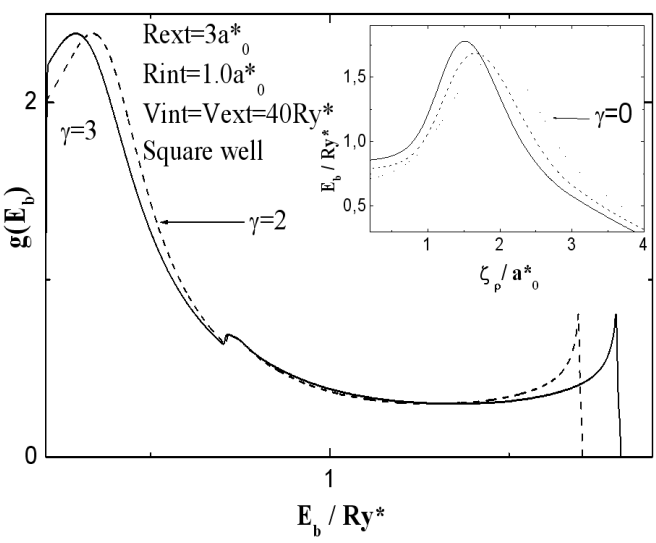

FIG. 3: Density of the $D^{0}$ impurity states as a function of the binding energy in a Nanotube for different values of the magnetic field $B$ applied parallel to the axis, Inset shows the corresponding donor binding energies dependencies on the distance from the axis.

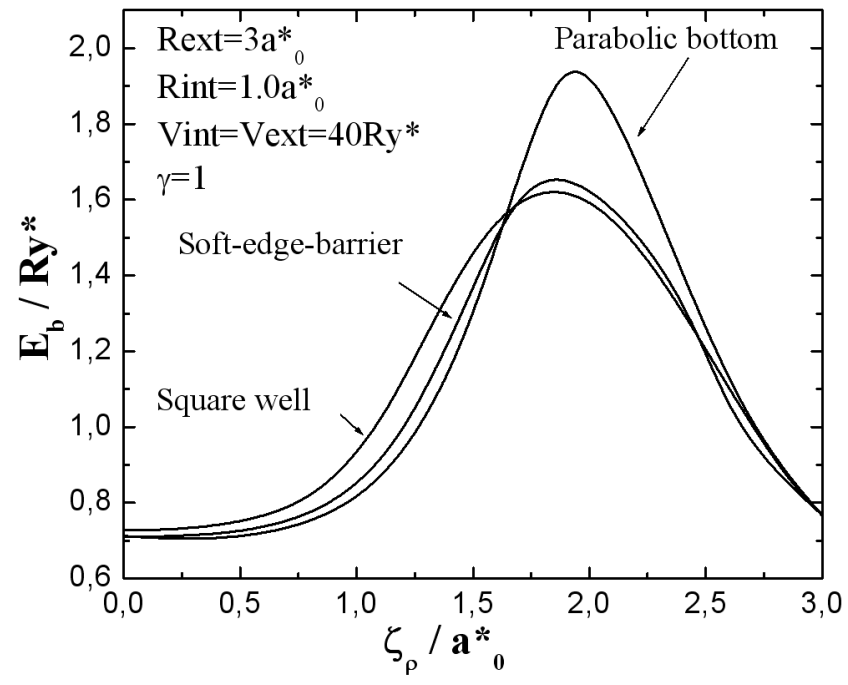

FIG. 4: $D^{0}$ ground state binding energies as a function of the donor displacement $\xi_{\rho}$ from the axis of the cylindrical nanotube for different potential shapes.

situated in the same position the electron-donor separations becomes very small. When a magnetic field is applied along of axial direction one can observe a successive displacement of the curve peaks position with increase of the strength magnetic field. This displacement is caused because the magnetic field provides additional confinements that tend to move the electron to the central region of the nanotube. Such behavior of the donor binding energies is also reflected in the curves of density of impurity states in nantube. It is seen from Fig. 3 that 
the repulsive core causes the appearance of the singularities near the right-side and left-side threshold of the curves of the density of the impurity states and it middle part. These singularities are due to the existence of one minimum and one maximum in the binding energies dependence where the derivate $d E\left(\varsigma_{\rho}\right) / d \varsigma_{\rho}$ is equal to zero.

In Fig. 4 , we present the $D^{0}$ binding energy dependence on the donor displacement from the center of the nantube using different potential shapes, from the square well potential to parabolic bottom potential. The effect of the smoothing of the confinement potential is similar to the increasing of the magnetic field, but it is not as pronounced as in the case of the magnetic field.

\section{CONCLUSION}

We have presented a simple method of calculation of the binding energy for the lowest state of the off-axis $D^{0}$ donors in a nanotube with different potential shapes in the presence of the magnetic field applied parallel to the axis, taking into account the effect of the mixing $s$ y $p_{x}$ subbands. We found that under external magnetic field applied parallel to the axis the binding energies of donors located close to the axis increases and located far from the axis decreases, whereas the binding energies of donors distant from the interior barrier of nanotube about one effective Bohr radius are almost insensitive to the external magnetic field.

Also we analyze the density of the $D^{0}$ impurity states in quantum well wires for different magnetic fields

\section{Acknowledgement}

This work was partially financed by the Universidad Autónoma de Bucaramanga (UNAB) and the Universidad Santo Tomas (USTA).
[1] A. Fereira da Silva, Phys. Rev. B 41, 1684 (1990); A. Latge, N.Porras-Montenegro and L. E. Oliveira, Phys. Rev., B 45, 9420 (1992); L. E. Oliveira, N.Porras-Montenegro, and A. Latge, Phys. Rev. B 47, 13864 (1993); P. Villamizal and N. PorrasMontenegro, J. Phys.: Condens. Matter 10, 10599 (1998);

[2] S. V. Branis, G. Li and K. K. Bajaj, Phys. Rev. B 47, 1316 (1993); J. Cen and K. K Bajaj, Phys. Rev. B 46, 13280 (1992); J. Cen, S. M. Lee and K. K Bajaj, J. Appl. Phys. 73, 2848 (1993)

[3] F. J. Betancur, E. A. Orozco, J. D. Gonzalez, and I. D. Mikhailov,
Phys. Stat. Sol. 242 (b), 1833 (2005)

[4] J. Sierra-Ortega, I. D. Mikhailov, and F.J. Betancur, Physica B 348, 66 (2004)

[5] F. J. Betancur, I. D. Mikhailov, and L. E. Oliveira, J. Appl. Phys. D 31, 3391 (1998)

[6] I. D. Mikhailov, F. J. Betancur, R. Escorcia, and J. Sierra-Ortega, Phys. Stat. Sol. 234 (b), 590 (2002), Phys. Rev., B67, 156317 (2003) 